\title{
Autonomic arousal and experimentally induced pain: A critical review of the literature
}

\author{
Brandon N Kyle $\mathrm{PhD}^{1,2}$, Daniel W McNeil PhD ${ }^{2}$
}

BN Kyle, DW McNeil. Autonomic arousal and experimentally induced pain: A critical review of the literature. Pain Res Manag 2014;19(3):159-167.

BACKGROUND: Autonomic arousal frequently is assumed to be a component of the pain response, perhaps because physiological mechanisms connecting pain and autonomic reactivity can be easily conceptualized. The evidence clarifying autonomic responses specific to painful stimulation, however, has been rather sporadic and lacks coherence; thus, a summary and critical review is needed in this area.

OBJECTIVES: To summarize and integrate findings from 39 experimental investigations from 1970 to 2012 of pain-induced autonomic arousal in humans.

METHODS: Medline and PsycINFO databases were searched for relevant articles. References from these articles were also considered for review.

RESULTS: Painful stimuli increase respiration rate, induce muscle tension, intensify electrodermal activity and dilate the pupils. Cardiovascular activity also increases, but the pattern displayed in response to pain is complex; peripheral vasoconstriction and sympathetically mediated cardiac responses are most typical. Additionally, autonomic expression of pain shows inconsistent relations with verbal and overt motor responses.

CONCLUSIONS: Autonomic arousal can be legitimately measured and modified as one facet of the pain response. Future research should particularly focus on increasing sample size and broadening the diversity of participants. To improve the ability to compare and contrast findings across studies, as well as to increase the applicability of laboratory findings to naturalistic pain, investigators also must enhance experimental design by increasing uniformity or accounting for differences in methodology. Finally, further work remains to utilize more specific assessments of autonomic response and to assess relationships of autonomic reactivity with other cognitive (eg, attention) and affective (eg, anxiety) variables.

Key Words: Autonomic arousal; Pain; Physiological arousal

$\mathrm{T}$ heoretically, pain can be understood as a subjective, unpleasant experience with both sensory and emotional components (1). Adaptive and natural, acute pain signals a short-term somatic insult that can be addressed in multiple ways based on context $(2,3)$. Since the middle of the 20th century, laboratory-based investigations of experimentally induced pain have increased in number, but such research also has produced skepticism regarding external validity, generalizability and ability to accurately simulate the private experience of pain (4-9). Studying experimentally induced acute pain, however, offers several advantages: control over the physical properties of the stimulus (eg, magnitude, duration), manipulation of related variables and ability to relate quantifiable levels of manipulated variables to levels of participant responding $(6,9)$. In assessment, pain can be conceptualized pragmatically as a construct that can be measured in multiple response modalities: verbal report, overt motor behaviour and physiological activity $(10,11)$. Of the different types of physiological measurements, activity of the two subdivisions of the autonomic nervous system (ANS) - the sympathetic and the parasympathetic nervous systems - are particularly associated with pain $(6,12,13)$. Increased activity in the sympathetic nervous system leads to increases in sweating (electrodermal activity), respiration rate, heart rate, blood pressure,

\section{L'éveil autonome et la douleur induite de manière artificielle : une analyse bibliographique critique}

HISTORIQUE : On présume souvent que l'éveil autonome est un élément de la réponse à la douleur, peut-être parce que des mécanismes physiologiques qui relient la douleur et la réactivité autonome peuvent être plutôt complexes. Les données probantes permettant de clarifier les réponses autonomes propres à la stimulation douloureuse sont toutefois plutôt sporadiques et manquent de cohérence. C'est pourquoi un résumé et une analyse critique s'imposent dans ce domaine.

OBJECTIFS : Résumer et intégrer les résultats de 39 recherches expérimentales menées entre 1970 et 2012 sur l'éveil autonome induit par la douleur chez les humains.

MÉTHODOLOGIE : Les chercheurs ont dépouillé les bases de données Medline et PsycINFO pour trouver des articles pertinents. Ils ont également examiné les références de ces articles en vue de l'analyse.

RÉSULTATS : Les stimuli douloureux accroissent le rythme respiratoire, induisent une tension musculaire, intensifient l'activité électrodermique et dilatent les pupilles. L'activité cardiovasculaire augmente également, mais le schéma présenté en réponse à la douleur est complexe. La vasoconstriction périphérique et les réponses cardiaques à médiation sympathique sont très classiques. De plus, les relations entre l'expression autonome de la douleur et les réponses verbales et motrices évidentes sont inégales.

CONCLUSIONS : L'éveil autonome peut être mesuré et modifié de manière légitime comme une facette de la réponse à la douleur. Les prochaines recherches devraient s'attacher tout particulièrement à accroître la taille de l'échantillon et à élargir la diversité des participants. Pour mieux comparer et contraster les résultats entre les études et pour accroître l'applicabilité des résultats de laboratoire à la douleur naturelle, les chercheurs doivent également améliorer leur méthodologie expérimentale en favorisant l'uniformité ou en tenant compte des différences de méthodologie. Enfin, d'autres travaux devront faire appel à des évaluations plus précises de la réponse autonome et évaluer les relations à la réactivité autonome avec d'autres variables cognitives (p. ex., attention) et affectives (p. ex., anxiété).

vascular vasoconstriction and pupil dilation. Although parasympathetic activation generally results in responses opposite those of the sympathetic nervous system, the two branches can have more complex and ongoing interactions, particularly regarding cardiac activity (12). For example, although parasympathetic influence is typically the dominant cardiac influence and mediates decreased heart rate variability (ie, changes in the interval between heart beats) coupled with increased heart rate, increased heart rate due to diminished cardiac pre-ejection period (ie, the interval between ventricle contractions) is more sympathetically mediated $(12,14,15)$.

Despite recognition of the need to assess pain across modalities $(7,8,16)$, pain remains difficult to measure, particularly from a psychophysiological perspective (17). Perhaps because researchers can readily describe how physiological mechanisms should associate pain with autonomic arousal, investigators have long noted connections between pain and autonomic arousal $(6,14,18-20)$. In an early review of pain and autonomic arousal published several decades ago (6), pain was noted to quicken respiration rate, and increase blood flow to muscles, muscle tension, blood pressure, pulse rate, stroke volume and peripheral vasoconstriction. Difficulties with assessing a stimulus-specific autonomic response to pain were also noted (6), such as individual

${ }^{1}$ East Carolina University, Greenville, North Carolina; ${ }^{2}$ West Virginia University, Morgantown, West Virginia, USA

Correspondence: Dr Brandon N Kyle, Department of Psychiatric Medicine, Brody School of Medicine, 600 Moye Boulevard, Mail Stop 694,

East Carolina University, Greenville, North Carolina 27834-4354, USA. Telephone 252-744-4234, fax 252-744-2419,

e-mail kyleb@ecu.edu 
response stereotypy (14) and the law of initial values (21). Similarly, a more recent review of the extant literature for infants and young children by Sweet and McGrath (22) has suggested that pain in this age group increases heart rate, blood pressure and skin conductance, and decreases vagal tone.

\section{PURPOSE AND ORGANIZATION OF ARTICLE}

Since the 1960s, technology for autonomic assessment has advanced $(14,17)$. Nevertheless, research in the past several decades dedicated to clarifying the autonomic response to laboratory-induced acute pain - beyond superficial assumptions of a relationship - has been sporadic and lacks coherence.

The purpose of the current review is to promote a clearer understanding of the physiological component of the acute pain response in humans by critically examining empirical studies measuring autonomic response to experimentally induced painful stimulation. In addition to clarifying which autonomic responses are specific to pain, the review also examines the relationships between autonomic responses and responses in other measurement modalities.

To assemble literature, Medline and PsycINFO databases were searched (key words: 'pain', 'autonomic arousal', 'physiological arousal'). References from resultant articles were considered for review as well. The present review included only articles in which autonomic arousal was measured either as a change from baseline or as a form of comparison across groups in response to a laboratory-induced stimulus clearly identified as painful. Thus, the following studies were excluded: studies measuring autonomic arousal as a response to chronic or naturally occurring acute pain; studies that did not explicitly characterize stimulation as painful (eg, not merely stimulating nerve fibers associated with nociceptive reflexes); and studies not clearly measuring autonomic activity at the time of painful stimulation, in response to the stimulation, as a change from baseline or group comparison. Additionally, investigations of arousal affecting pain responding represent a separate line of research. Furthermore, based on literature suggesting general developmental differences between children and adults regarding pain (16), the review primarily focused on studies of adults. Although studies with children were not necessarily excluded, no article included in the review involved participants younger than eight years of age, and studies in the review by Sweet and McGrath (22) were not included. These inclusion and exclusion criteria were used to set a reasonable scope for a single review, to reduce ambiguity in interpretation, and to minimize experimental design differences that may limit comparison and contrast across studies. Finally, although controlled by the somatic nervous system, muscle tension was assessed as a correlate of autonomic arousal in several experiments (23) and was included in the review as a complementary component of the ANS in the peripheral nervous system.

Ultimately, 39 articles, published between 1970 and 2012, were identified for inclusion. Organized according to method of pain induction, the results of studies selected are briefly reviewed below and summarized in Tables 1 to 5. Studies including cold pressor stimulation typically involved immersion of participants' hands or feet into a bath of ice water, while studies examining heat stimulation used hot water for immersion or heat thermodes placed on participants' skin. Investigations of pressure stimulation typically used an algometer (24-26) applied to participants' fingers to produce a slowly building pressure sensation, while research using electrical current often presented stimulation to the extremities or face for durations of seconds or less. Finally, studies relying on other, less common methods of pain induction were grouped separately. Following the brief review, an overall critique of strengths and weaknesses across the literature is presented, along with implications and recommendations for future research.

\section{Cold-pressor stimulation}

\section{REVIEW OF THE LITERATURE}

Significant increases in electrodermal activity and respiratory rate have been reported in response to cold-pressor stimulation compared with baseline and guided imagery (27). Skin conductance response to cold-pressor stimulation was also increased by greater levels of trait anxiety and was decreased by the social influence of reassurance from an in-group member confederate $(28,29)$. Electrodermal activity, however, was discordant from cold-pressor tolerance (ie, interval until escape $[28,29])$, possibly because many participants endured the cold pressor for the maximum time, reducing variability in tolerance (29).

Across the lifespan, cold pressor stimulation increased cardiovascular response when compared with baseline (27,30-33), warm pressor (34) and vicarious experience (35). Additionally, imagery-based distraction (28) and odourant administration (36) diminished vasoconstriction and blood pressure responses to cold pressor while simultaneously increasing pain tolerance and decreasing pain report, although greater sensory input from odourant administration may have increased heart rate. Findings for cardiovascular response were not uniform, however, and nonsignificant or discordant results were reported across measures and studies (27,28,31,33,34,36-39). Inconsistencies in cardiovascular findings may be attributable to imprecise blood pressure and cardiac recordings obscuring the relative contributions of sympathetic and parasympathetic influence.

Pupil dilation increased in the first $30 \mathrm{~s}$ after cold pressor administration and decreased in response to a hypnosis intervention, but pupil dilation was not correlated either with cardiovascular responses or verbal report of pain $(33,40)$. Finally, although studies examining muscle tension in response to cold pressor stimuli produced conflicting results, the use of a less intense cold pressor stimulus or the use of pleasant and relaxing imagery $(27,28)$ may explain discrepancies from studies producing significant results $(30,31)$.

\section{Algometer pressure stimulation}

Although electrodermal activity, respiration rate and cardiovascular activity (with decreased heart rate variability) all increased with algometer pressure stimulation $(23,41,42)$, only respiration rate, of the three, appeared to characterize a stimulus-specific response $(23,43,44)$. None of these three domains of ANS activity, however, was correlated with pain report within subjects (23). Regarding group differences, skin conductance response fluctuations, but not respiration rate or heart rate, were greater in patients with back pain compared with healthy controls (23); electrodermal activity did not differ for women with and without premenstrual syndrome (41).

Pupil dilation, although not tested directly for a stimulus-specific response, increased with increasing levels of algometer pressure stimulation intensity and correlated with pain report $(45,46)$. Muscle tension also increased in response to algometer pressure and differentiated response to pain from response to slides of homicide victims $(4,43)$; nonsignificant results for muscle tension in a study by Peters and Schmidt (23) may be attributable to analysis of muscle tension data from only the initial portion of the study, compared with the entirety of the experiment.

\section{Electrical stimulation}

Increased electrodermal activity, cardiovascular activity (particularly heart rate) and pupil dilation with higher electrical shock intensity was consistent across studies (47-53). Furthermore, the autonomic response to electrical stimulation was modifiable. When participants were able to predict the timing of electrical stimulation, paired with a confederate or model displaying tolerance of shock, and engaged in a music listening task, skin conductance, heart rate reactivity and pupil dilation decreased $(47,49,50,52)$. Additionally, when greater shock intensity was used, electrodermal activity and heart rate matched verbal reports of pain (50-52).

Additionally, one study reviewed examined the influence of shock intensity on an index of arousal created from combining skin conductance, heart rate and pupil dilation into a single variable (54). Although men accepted higher stimulus intensities and had higher arousal levels than women, the two sexes did not differ on reported pain level and accuracy (ie, concordance between pain rating and stimulus intensity), but men were more accurate with increased arousal. 
TABLE 1

Sample size, sample sex, autonomic nervous system (ANS) measures, pain stimuli details and main findings of cold pressor studies reviewed

\begin{tabular}{|c|c|c|c|c|c|}
\hline $\begin{array}{l}\text { Author(s) } \\
\text { (reference), year }\end{array}$ & $\mathbf{n}$ & Female, \% & ANS measure(s) & Pain stimulus & Main ANS findings \\
\hline $\begin{array}{l}\text { Yamaguchi et al } \\
\text { (35), } 1978\end{array}$ & 40 & 25 & $\begin{array}{l}\text { Pulse amplitude, heart } \\
\text { rate }\end{array}$ & $\begin{array}{l}\text { Ice bar gripped } 5 \text { times for } \\
30 \mathrm{~s}\end{array}$ & $\begin{array}{l}\text { Compared with vicarious experience, vasoconstriction and heart } \\
\text { rate increased during direct experience of the cold pressor }\end{array}$ \\
\hline $\begin{array}{l}\text { Feuerstein et al } \\
(30), 1982\end{array}$ & 29 & 58.6 & $\begin{array}{l}\text { Digital blood volume } \\
\text { pulse, heart rate, } \\
\text { muscle tension }\end{array}$ & Cold pressor at $0^{\circ} \mathrm{C}$ for $60 \mathrm{~s}$ & $\begin{array}{l}\text { Vasoconstriction, heart rate and muscle tension increased during } \\
\text { cold pressor, especially during the first } 30 \mathrm{~s}\end{array}$ \\
\hline $\begin{array}{l}\text { Peckerman et al } \\
\text { (32), } 1991\end{array}$ & 18 & 0 & SBP, DBP, heart rate & $\begin{array}{l}\text { Cold pressor at } 1^{\circ} \mathrm{C} 3 \text { times, } \\
\text { endured for } 180 \mathrm{~s}\end{array}$ & $\begin{array}{l}\text { Blood pressure increased up to } 150 \mathrm{~s} \text { after stimulation; heart rate } \\
\text { increased for } 30 \mathrm{~s} \text {, then increased again between } 90 \mathrm{~s} \text { and } 150 \mathrm{~s}\end{array}$ \\
\hline $\begin{array}{l}\text { Biederman and } \\
\text { Schefft (28), } \\
1994\end{array}$ & 46 & 100 & $\begin{array}{l}\text { Skin conductance level, } \\
\text { skin temperature, SBP, } \\
\text { DBP, muscle tension }\end{array}$ & $\begin{array}{l}\text { Cold pressor at } 0^{\circ} \mathrm{C} \text { twice } \\
\text { until tolerance }\end{array}$ & $\begin{array}{l}\text { During the second cold pressor test, the skin conductance level of } \\
\text { participants with greater trait anxiety increased, while imagery- } \\
\text { based distraction decreased skin temperature and muscle tension }\end{array}$ \\
\hline $\begin{array}{l}\text { Tassorelli et al } \\
\text { (33), } 1995\end{array}$ & 10 & 50 & $\begin{array}{l}\text { Heart rate, mean blood } \\
\text { pressure, pupil dilation }\end{array}$ & $\begin{array}{l}\text { Cold pressor of } 4^{\circ} \mathrm{C} \text { endured } \\
\text { for } 300 \mathrm{~s}\end{array}$ & $\begin{array}{l}\text { Pupils dilated and blood pressure increased } 30 \mathrm{~s} \text { and } 120 \mathrm{~s} \text {, } \\
\text { respectively, after cold pressor onset }\end{array}$ \\
\hline $\begin{array}{l}\text { Tomaka et al } \\
(34), 1997\end{array}$ & 43 & NR & $\begin{array}{l}\text { Total peripheral } \\
\text { resistance, pre-ejection } \\
\text { period, heart rate, } \\
\text { cardiac output }\end{array}$ & $\begin{array}{l}\text { Cold pressor of } 1^{\circ} \mathrm{C} \text { endured } \\
\text { for } 180 \mathrm{~s}\end{array}$ & $\begin{array}{l}\text { Vasoconstriction increased during cold pressor when compared } \\
\text { with warm pressor }\end{array}$ \\
\hline $\begin{array}{l}\text { Zeichner et al } \\
\text { (66), } 1999\end{array}$ & 42 & NR & Pulse rate & $\begin{array}{l}\text { Cold pressor of } 0^{\circ} \mathrm{C}-4^{\circ} \mathrm{C} \\
\text { endured for } 180 \mathrm{~s}\end{array}$ & $\begin{array}{l}\text { Participants with less exposure to family pain modelling displayed } \\
\text { increased pulse rate during cold pressor }\end{array}$ \\
\hline $\begin{array}{l}\text { Zeichner et al } \\
\text { (39), } 2000\end{array}$ & 42 & 57.1 & Pulse rate & $\begin{array}{l}\text { Cold pressor of } 0^{\circ} \mathrm{C}-4^{\circ} \mathrm{C} \\
\text { endured for } 120 \mathrm{~s}\end{array}$ & Women had increased pulse rate during cold pressor test \\
\hline $\begin{array}{l}\text { Myers et al (38), } \\
2001\end{array}$ & 104 & 48.1 & SBP, DBP, heart rate & $\begin{array}{l}\text { Cold pressor of } 1^{\circ} \mathrm{C}-3^{\circ} \mathrm{C} \\
\text { endured until tolerance }\end{array}$ & $\begin{array}{l}\text { Baseline SBP was correlated with pain tolerance. Males had higher } \\
\text { heart rate change scores }\end{array}$ \\
\hline $\begin{array}{l}\text { James and } \\
\text { Hardardottir } \\
\text { (31), } 2002\end{array}$ & 72 & 50 & $\begin{array}{l}\text { SBP, DBP, heart rate, } \\
\text { muscle tension }\end{array}$ & $\begin{array}{l}\text { Cold pressor at } 2^{\circ} \mathrm{C} \text { delivered } \\
\text { twice until tolerance }\end{array}$ & $\begin{array}{l}\text { SBP, DBP and heart rate increased during cold pressor. Muscle } \\
\text { tension increased over the course of the experiment }\end{array}$ \\
\hline $\begin{array}{l}\text { Flora et al (37), } \\
2003\end{array}$ & 34 & 82.4 & SBP, DBP, pulse rate & $\begin{array}{l}\text { Cold pressor at } 5^{\circ} \mathrm{C} \text { delivered } \\
\text { until tolerance }\end{array}$ & $\begin{array}{l}\text { Pulse rate decreased for participants enduring the cold pressor } \\
\text { during a self-control procedure, but not for participants enduring a } \\
\text { warm pressor }\end{array}$ \\
\hline $\begin{array}{l}\text { Foster et al (27), } \\
2003\end{array}$ & 100 & 47.0 & $\begin{array}{l}\text { Skin conductance activity, } \\
\text { respiration rate, skin } \\
\text { temperature, SBP, DBP, } \\
\text { heart rate, muscle } \\
\text { tension }\end{array}$ & Cold pressor at $13^{\circ} \mathrm{C}$ for $40 \mathrm{~s}$ & $\begin{array}{l}\text { Skin conductance, respiration rate, SBP and DBP increased during } \\
\text { painful stimulation relative to baseline and guided imagery }\end{array}$ \\
\hline $\begin{array}{l}\text { Raudenbush } \\
\text { et al (36), } 2004\end{array}$ & 158 & 50 & $\begin{array}{l}\mathrm{O}_{2} \text { saturation, } \mathrm{SBP}, \mathrm{DBP}, \\
\text { heart rate }\end{array}$ & $\begin{array}{l}\text { Cold pressor of } 3^{\circ} \mathrm{C} \text { delivered } \\
\text { until tolerance }\end{array}$ & $\begin{array}{l}\text { Pleasant odours decreased SBP and DBP and increased } \mathrm{O}_{2} \\
\text { saturation during cold pressor }\end{array}$ \\
\hline $\begin{array}{l}\text { Walter et al (40), } \\
2005\end{array}$ & 22 & 50 & Pupil dilation & $\begin{array}{l}\text { Ice spray of }-50^{\circ} \mathrm{C} \text { delivered } \\
\text { twice for } 0.5 \mathrm{~s}\end{array}$ & $\begin{array}{l}\text { Maximal pupil dilation and absolute pupil diameter in response to } \\
\text { cold pressor were both less during hypnotic state than during } \\
\text { nonhypnotic state }\end{array}$ \\
\hline $\begin{array}{l}\text { Platow et al (29), } \\
2007\end{array}$ & 54 & 40.7 & Galvanic skin response & $\begin{array}{l}\text { Cold pressor of } 0^{\circ} \mathrm{C}-2^{\circ} \mathrm{C} \\
\text { delivered twice and endured } \\
\text { to tolerance }\end{array}$ & $\begin{array}{l}\text { Skin conductance decreased during the second cold pressor trial } \\
\text { for participants receiving reassurance from an in-group member }\end{array}$ \\
\hline
\end{tabular}

DBP Diastolic blood pressure; NR Not reported; SBP Systolic blood pressure

\section{Heat stimulation}

Although studies investigating heat stimulation examined autonomic activity using only electrodermal and cardiovascular activity, responding in both ANS domains increased with painful heat (55-57). The use of more precise cardiac assessments suggested that increased cardiac response was due to enhanced sympathetic activity and diminished parasympathetic activity, which also appeared to drive decreased heart rate with placebo administration $(55,57,58)$. Additionally, heart rate was responsive to hypnotic instructions to alter pain unpleasantness (59).

Electrodermal and cardiovascular activity diverged, however, in their concordance with other response modalities. Male participants exhibited greater skin conductance levels but reported less pain and arousal, and correlations between skin conductance level and participant report failed to reach statistical significance, perhaps because of insufficient power (55). In contrast, the changes in cardiovascular activity were closely related to report of pain across studies, particularly for males $(55,57)$.
Other stimuli

Three additional studies examined other methods of pain induction. Although heart rate response did not increase with ascorbic acid injection compared with saline injection (60), injection of glutamate into myofascial trigger points produced vasoconstriction comparable with that produced by maximum inspiratory breath hold, which is known to sympathetically increase vasoconstriction (61). Finally, in a study of placebo analgesia, heart-rate response was related to painful stimulation; although there was concordance between pain report and heart-rate response following the administration of saline, atropine and naloxone, propanolol reduced pain reporting but not heartrate response (62).

\section{CRITIQUE OF THE LITERATURE REVIEWED}

Sample issues

The frequent use of college students willing to endure experimentally induced pain places a limit on the scope of generalization of the 
TABLE 2

Sample size, sample sex, autonomic nervous system (ANS) measures, pain stimuli details and main findings of algometer pressure pain studies reviewed

\begin{tabular}{|c|c|c|c|c|c|}
\hline $\begin{array}{l}\text { Author(s) } \\
\text { (reference), year }\end{array}$ & $\mathbf{n}$ & Female, \% & ANS measure(s) & Pain stimulus & Main ANS findings \\
\hline $\begin{array}{l}\text { Davidson and } \\
\text { Neufeld (43), } 1974\end{array}$ & 60 & 100 & $\begin{array}{l}\text { Skin conductance } \\
\text { response, respiration } \\
\text { rate, heart rate, } \\
\text { muscle tension }\end{array}$ & $\begin{array}{l}\text { Algometer pressure for } 5 \text { trials at } \\
\text { threshold and } 5 \text { trials within } \\
0.2 \mathrm{~g} \text { of tolerance }\end{array}$ & $\begin{array}{l}\text { Increased respiratory rate and increased muscle tension } \\
\text { differentiated pain from a psychological stressor }\end{array}$ \\
\hline Linden (44), 1985 & 60 & 53.33 & SBP, DBP, heart rate & $\begin{array}{l}\text { Algometer pressure of } 400 \mathrm{~g} \\
\text { delivered for } 180 \mathrm{~s}\end{array}$ & $\begin{array}{l}\text { Heart rate, SBP and DBP increased less during algometer } \\
\text { stimulation when compared with mental arithmetic and } \\
\text { projective testing }\end{array}$ \\
\hline $\begin{array}{l}\text { Kuczmierczyk and } \\
\text { Adams (41), } 1986\end{array}$ & 21 & 100 & $\begin{array}{l}\text { Skin conductance } \\
\text { level, heart rate }\end{array}$ & $\begin{array}{l}\text { Algometer pressure of } 2000 \mathrm{~g} \\
\text { endured twice until tolerance }\end{array}$ & $\begin{array}{l}\text { Skin conductance level and heart rate increased at pain } \\
\text { threshold across menstrual cycle phases }\end{array}$ \\
\hline $\begin{array}{l}\text { Peters and Schmidt } \\
\text { (23), } 1991\end{array}$ & 40 & 0 & $\begin{array}{l}\text { Skin conductance } \\
\text { level and response, } \\
\text { respiration rate, } \\
\text { heart rate, muscle } \\
\text { tension }\end{array}$ & $\begin{array}{l}\text { Algometer pressure of } 1700 \mathrm{~g} \text {, } \\
\text { delivered } 6 \text { times, endured for } \\
70 \% \text { of tolerance time }\end{array}$ & $\begin{array}{l}\text { Skin conductance response fluctuations, respiration rate and } \\
\text { heart rate increased with pressure stimulation, while heart rate } \\
\text { variability decreased }\end{array}$ \\
\hline $\begin{array}{l}\text { Ellermeier and } \\
\text { Westphal (45), } \\
1995\end{array}$ & 16 & 50 & Pupil dilation & $\begin{array}{l}\text { Algometer pressure of } 750 \mathrm{kPa} \text {, } \\
940 \mathrm{kPa}, 1190 \mathrm{kPa} \text { and } 1500 \mathrm{kPa} \\
\text { delivered four times each for } 20 \mathrm{~s}\end{array}$ & Pupil dilation increased with increased pressure intensity \\
\hline Carter et al (4), 2002 & 80 & 50 & $\begin{array}{l}\text { Heart rate, muscle } \\
\text { tension }\end{array}$ & $\begin{array}{l}\text { Algometer pressure, delivered } \\
\text { twice, until tolerance }\end{array}$ & $\begin{array}{l}\text { Heart rate decreased from the first algometer trial to the second. } \\
\text { Trapezius muscle tension increased during algometer stimulation. }\end{array}$ \\
\hline $\begin{array}{l}\text { Höfle et al (46), } \\
2008\end{array}$ & 20 & 100 & Pupil dilation & $\begin{array}{l}\text { Algometer pressure of } 800 \mathrm{~g} \text { and } \\
950 \mathrm{~g} \text {, delivered six times each } \\
\text { for } 20 \mathrm{~s}\end{array}$ & $\begin{array}{l}\text { Pupil dilation was greater } 10 \mathrm{~s} \text { to } 20 \mathrm{~s} \text { after the onset of the } \\
950 \mathrm{~g} \text { stimulus than the } 800 \mathrm{~g} \text { stimulus }\end{array}$ \\
\hline Kyle et al (42), 2009 & 96 & 50 & Heart rate & $\begin{array}{l}\text { Algometer pressure of } 1000 \mathrm{~g} \\
\text { delivered twice and } 2000 \mathrm{~g} \\
\text { delivered once until tolerance }\end{array}$ & $\begin{array}{l}\text { Heart rate was higher during the } 2000 \mathrm{~g} \text { stimulus, compared with } \\
\text { the } 1000 \mathrm{~g} \text { stimulus, but only when the } 2000 \mathrm{~g} \text { was delivered } \\
\text { first }\end{array}$ \\
\hline
\end{tabular}

DBP Diastolic blood pressure; SBP Systolic blood pressure

\section{TABLE 3}

Sample size, sample sex, autonomic nervous system (ANS) measures, pain stimuli details and main findings of electrical stimulation studies reviewed

\begin{tabular}{|c|c|c|c|c|c|}
\hline $\begin{array}{l}\text { Author(s) } \\
\text { (reference), year }\end{array}$ & $\mathbf{n}$ & Female, \% & ANS measure(s) & Pain stimulus & Main ANS findings \\
\hline $\begin{array}{l}\text { Lykken et al (52), } \\
1972\end{array}$ & 48 & 0 & $\begin{array}{l}\text { Skin conductance level, } \\
\text { heart rate }\end{array}$ & $\begin{array}{l}\text { Electric current, } 0.5 \mathrm{~s} \text { in duration, delivered } \\
48 \text { times at tolerance }\end{array}$ & $\begin{array}{l}\text { Skin conductance and heart rate were lower when } \\
\text { participants could predict the timing, but not } \\
\text { location, of electrical stimulation }\end{array}$ \\
\hline $\begin{array}{l}\text { Craig and } \\
\text { Neidermayer (49), } \\
1974\end{array}$ & 40 & 0 & $\begin{array}{l}\text { Skin conductance level, } \\
\text { heart rate }\end{array}$ & $\begin{array}{l}\text { Electric current, } 0.5 \mathrm{~s} \text { in duration, } \\
\text { increasing in intensity to threshold }\end{array}$ & $\begin{array}{l}\text { Skin conductance level and heart rate increased } \\
\text { across groups with electrical stimulation }\end{array}$ \\
\hline $\begin{array}{l}\text { Lanzetta et al (51), } \\
1976\end{array}$ & 20 & 50 & $\begin{array}{l}\text { Skin conductance } \\
\text { response }\end{array}$ & $\begin{array}{l}\text { Electric current, } 2 \text { s in duration, delivered } \\
41 \text { times at intensities of } 33 \%, 66 \% \text { and } \\
99 \% \text { of tolerance }\end{array}$ & $\begin{array}{l}\text { Electrodermal response increased with increased } \\
\text { electrical stimulation intensity }\end{array}$ \\
\hline $\begin{array}{l}\text { Craig and Prkachin } \\
\text { (50), } 1978\end{array}$ & 20 & 100 & $\begin{array}{l}\text { Skin conductance } \\
\text { response, skin potential } \\
\text { response, heart rate }\end{array}$ & $\begin{array}{l}\text { Electric current, } 0.5 \mathrm{~s} \text { in duration, } \\
\text { increasing in intensity to threshold, } \\
\text { followed by } 12 \text { presentations each of } \\
2 \mathrm{~mA}, 3 \mathrm{~mA}, 4 \mathrm{~mA}, 5 \mathrm{~mA} \text { and } 6 \mathrm{~mA}\end{array}$ & $\begin{array}{l}\text { Skin conductance response increased during } \\
\text { stimulation. Heart rate increased after electrical } \\
\text { shock, particularly with elevated shock intensity }\end{array}$ \\
\hline $\begin{array}{l}\text { Chapman et al (48), } \\
1999\end{array}$ & 20 & 45 & Pupil dilation & $\begin{array}{l}\text { Electric current, } 5 \mathrm{~ms} \text { in duration, at four } \\
\text { individualized intensities, given in } 2 \text { blocks } \\
\text { of } 64\end{array}$ & $\begin{array}{l}\text { Pupil dilation increased as stimulus magnitude } \\
\text { increased. Peak pupil dilation occurred later with } \\
\text { increasing stimulus intensity }\end{array}$ \\
\hline $\begin{array}{l}\text { Chapman et al (54), } \\
2002\end{array}$ & 100 & 44 & $\begin{array}{l}\text { Pupil dilation, skin } \\
\text { conductance response, } \\
\text { heart rate }\end{array}$ & $\begin{array}{l}\text { Electric current, } 5 \mathrm{~ms} \text { in duration, at three } \\
\text { individualized intensities, given in } 4 \text { blocks } \\
\text { of } 36\end{array}$ & $\begin{array}{l}\text { Increased stimulus intensity led to increased } \\
\text { attention to stimulus, which led to increased ANS } \\
\text { arousal }\end{array}$ \\
\hline $\begin{array}{l}\text { Vassend and } \\
\text { Knardahl (53), } \\
2005\end{array}$ & 58 & 100 & $\begin{array}{l}\text { Skin blood flow, mean } \\
\text { arterial pressure, heart } \\
\text { rate }\end{array}$ & $\begin{array}{l}\text { Electric current, delivered } 5 \text { times, } \\
\text { increasing in intensity up to tolerance }\end{array}$ & $\begin{array}{l}\text { Heart rate, mean arterial pressure, facial } \\
\text { vasodilation and digital finger vasoconstriction } \\
\text { increased during stimulation }\end{array}$ \\
\hline $\begin{array}{l}\text { Bradshaw et al (47), } \\
2012\end{array}$ & 49 & 39.6 & Pupil dilation & $\begin{array}{l}\text { Electric current, } 5 \text { ms in duration, delivered } \\
\text { in } 9 \text { blocks of } 24 \text {, at } 20 \%, 50 \% \text { and } 80 \% \\
\text { of individual tolerance }\end{array}$ & $\begin{array}{l}\text { Pupil dilation increased with increasing stimulus } \\
\text { intensity but decreased with increasing } \\
\text { complexity of a music listening task }\end{array}$ \\
\hline
\end{tabular}

39 studies reviewed (63). Only seven studies did not rely on participants from a traditional college student age range $(23,27,30,41,50,53,62)$. Overutilization of college-age students is especially problematic because many pain responses differ across the lifespan; for example, nervous system activity latency increases and amplitude decreases in older adults (16). How such differences impact autonomic response to 
TABLE 4

Sample size, sample sex, autonomic nervous system (ANS) measures, pain stimuli details and main findings of heat stimulus studies reviewed

\begin{tabular}{|c|c|c|c|c|c|}
\hline \multirow{2}{*}{$\begin{array}{l}\text { Author(s), year } \\
\text { (reference) }\end{array}$} & \multicolumn{3}{|c|}{ Female, } & \multirow[b]{2}{*}{ Pain stimulus } & \multirow[b]{2}{*}{ Main ANS findings } \\
\hline & $\mathbf{n}$ & $\%$ & ANS measure(s) & & \\
\hline $\begin{array}{l}\text { Möltner et al (56), } \\
1990\end{array}$ & 12 & 50 & $\begin{array}{l}\text { Respiration rate, heart } \\
\text { rate }\end{array}$ & $\begin{array}{l}\text { Heat thermode delivered } 2 \text { times at } \\
40^{\circ} \mathrm{C}, 2 \text { times at } 42^{\circ} \mathrm{C}, 2 \text { times at } 44^{\circ} \mathrm{C} \\
\text { and } 14 \text { times within } \pm 1.5^{\circ} \mathrm{C} \text { of pain } \\
\text { threshold }\end{array}$ & $\begin{array}{l}\text { Heat stimulation evoked monophasic and biphasic } \\
\text { increase in heart rate, and greater stimulus intensity } \\
\text { was associated with greater mean heart rate increase }\end{array}$ \\
\hline \multirow[t]{2}{*}{$\begin{array}{l}\text { Rainville et al } \\
\text { (59), } 1999\end{array}$} & Exp 1: 17 & 29.41 & Heart rate & $\begin{array}{l}\text { Hot pressor of } 46.5^{\circ} \mathrm{C} \text { delivered } 15 \text { times } \\
\text { for } 60 \mathrm{~s} \text { or up to tolerance }\end{array}$ & $\begin{array}{l}\text { Hypnosis instructions to decrease pain unpleasantness } \\
\text { decreased prestimulus resting heart rate }\end{array}$ \\
\hline & Exp 2: 20 & 35 & Heart rate & $\begin{array}{l}\text { Hot pressor of individualized intensity } \\
\text { delivered } 8 \text { times for } 60 \mathrm{~s}\end{array}$ & $\begin{array}{l}\text { Hypnosis instructions to decrease pain unpleasantness } \\
\text { decreased heart rate }\end{array}$ \\
\hline $\begin{array}{l}\text { Tousignant- } \\
\text { Laflamme et al } \\
(57), 2005\end{array}$ & 39 & 51.28 & $\begin{array}{l}\text { Skin conductance } \\
\text { level, heart rate }\end{array}$ & Hot water at $47^{\circ} \mathrm{C}$ for $120 \mathrm{~s}$ & $\begin{array}{l}\text { Skin conductance level and heart rate increased during } \\
\text { heat stimulation }\end{array}$ \\
\hline $\begin{array}{l}\text { Aslaksen et al } \\
\text { (55), } 2007\end{array}$ & 64 & 50 & $\begin{array}{l}\text { Skin conductance } \\
\text { level, heart rate }\end{array}$ & Heat thermode at $48^{\circ} \mathrm{C} 15$ times for $12 \mathrm{~s}$ & $\begin{array}{l}\text { Skin conductance level and heart rate increased with } \\
\text { stimulation }\end{array}$ \\
\hline $\begin{array}{l}\text { Aslaksen and } \\
\text { Flaten (58), } \\
2008\end{array}$ & 63 & 51 & Heart rate & Heat thermode at $46^{\circ} \mathrm{C} 10$ times for $240 \mathrm{~s}$ & $\begin{array}{l}\text { Placebo administration, relative to no placebo, } \\
\text { decreased heart rate }\end{array}$ \\
\hline
\end{tabular}

Exp Experiment

TABLE 5

Sample size, sample sex, autonomic nervous system (ANS) measures, pain stimuli details and main findings of other stimulus studies reviewed

\begin{tabular}{|c|c|c|c|c|c|}
\hline $\begin{array}{l}\text { Author(s), year } \\
\text { (reference) }\end{array}$ & $\mathbf{n}$ & $\begin{array}{c}\text { Female, } \\
\%\end{array}$ & ANS measure(s) & Pain stimulus & Main ANS findings \\
\hline \multirow[t]{2}{*}{$\begin{array}{l}\text { Pollo et al (62), } \\
2003\end{array}$} & Exp 1: 37 & 81.1 & Heart rate & $\begin{array}{l}\text { Electric current, delivered } 4 \text { times } \\
\text { at } 1.5 \text { times individual pain } \\
\text { threshold }\end{array}$ & $\begin{array}{l}\text { Placebo injection decreased heart rate during electrical } \\
\text { stimulation, relative to no placebo injection }\end{array}$ \\
\hline & $\operatorname{Exp} 2: 58$ & 53.5 & Heart rate & $\begin{array}{l}\text { Tonic, ischemic arm pain induced } \\
\text { via tourniquet procedure }\end{array}$ & $\begin{array}{l}\text { Placebo administration of saline and atropine produced a } \\
\text { decrease in heart rate. Naloxone and propanolol did not } \\
\text { decrease heart rate }\end{array}$ \\
\hline $\begin{array}{l}\text { Porro et al (60), } \\
2003\end{array}$ & 56 & 57.1 & Heart rate & Injection of ascorbic acid & $\begin{array}{l}\text { There were no differences in heart rate for injection of } \\
\text { ascorbic acid versus injection of saline or touching the } \\
\text { foot with a needle }\end{array}$ \\
\hline $\begin{array}{l}\text { Kimura et al (61), } \\
2009\end{array}$ & 14 & 35.7 & $\begin{array}{l}\text { Skin blood flow, skin } \\
\text { temperature }\end{array}$ & $\begin{array}{l}\text { Injection of } 0.1 \mathrm{~mL} \text { glutamate into } \\
\text { latent myofascial trigger points }\end{array}$ & $\begin{array}{l}\text { Injection of glutamate decreased skin blood flow and } \\
\text { temperature. Vasoconstriction was similar to maximum } \\
\text { inspiratory breath hold }\end{array}$ \\
\hline
\end{tabular}

\section{Exp Experiment}

experimentally induced painful stimulation across the lifespan remains uninvestigated.

Biological sex also emerged as a sampling issue. Ten studies recruited participants exclusively of one sex. Of the 29 studies involving participants of both sexes, only seven reported statistically significant differences according to sex in ANS pain response: males displayed increased cardiovascular reactivity and greater pain threshold and tolerance, responses that are more closely associated with report of pain than for females $(4,27,38,39,54,55,57)$. Previous research $(64,65)$, however, has suggested a minimum of 82 participants is needed for sufficient statistical power $(0.70)$ to detect sex differences in response to experimentally induced pain, and only five studies reviewed collected samples of this size or larger $(27,36,38,42,54)$. As such, the studies reviewed offered minimal opportunity to properly assess sex differences or similarities with adequate statistical power.

Addressing racial, ethnic and cultural similarities and differences also proved to be a significant weakness for many studies reviewed. Only five studies reviewed even reported the percentage of participants in different racial or ethnic categories $(4,27,32,38,42)$.

Procedure and design issues

Procedure issues: Studies using electric current typically presented shocks lasting seconds or less in duration, with ANS response often measured after stimulus offset. In contrast, cold pressor or pressure stimulation often occurred over the course of several minutes, and autonomic response was recorded during stimulation. The timing of pain during cold pressor (within $1 \mathrm{~min}$ ) and algometer (slowly building) stimuli differ as well $(6,24,25)$. Although such differences in duration and peak of noxious stimulation, as well as timing of autonomic assessment, make it more difficult to abstract generalizations or explain conflicting results for cardiovascular responding and muscle tension, patterns of responding that occurred similarly across different types of noxious stimuli for electrodermal activity, respiration and pupil dilation appear to be more robust.

Another issue limiting generalization across studies is the difference between individualized and uniform pain stimuli. Noxious stimulation alone does not necessarily equate with painful stimulation or produce pain responding, and investigators must balance attempts at uniformity in stimulation with individual differences in pain report, threshold and tolerance. Twelve studies reviewed changed the pain stimulus for each individual, which may reduce external validity and generalization to more natural contexts, as well as introduce bias from variation in accuracy of pain reporting $(23,43,47-52,54,56,59,62)$. Experiments with uniform stimuli that allowed participants to escape the noxious stimulus when they reached pain tolerance, however, created a confound of different stimulus durations $(4,28,29,31,36-38,41,42,53,59)$, and when the same stimulus was presented for the same duration, the stimulus was often of relatively reduced intensity or duration $(27,35,40,44-46,55)$. When studies used truly uniform and 
painful stimuli, they generally found significant results for autonomic response $(30,32-34,39,57,58,61,62,66)$.

Despite the potential influence of anxiety or fear of upcoming noxious stimulation, or the influence of being in a novel laboratory environment, only four studies reported waiting for stable autonomic activity before painful stimulation began $(27,31,38,61)$, with seven other studies including prestimulation adaptation periods lasting 10 min or longer $(33,35,41,44,45,49,51)$. Similarly, studies administering stimulation more than once differed from seconds to days in their interstimulus intervals. These vast differences in pre- and interstimulus adaptation periods impede interpretation across studies because the law of initial values suggests these levels influence later levels of arousal $(21,67)$.

A final noteworthy procedural limitation across studies involves the orienting and defensive responses. Disagreement exists regarding how best to conceptualize these responses because of equivocal results across investigations (68-70), providing an opportunity for investigations of autonomic response to laboratory-induced pain to contribute to this literature. Briefly, the orienting response enhances attention and sensitivity to moderate novel stimulation and involves cephalic vasodilation, peripheral vasodilation, increased electrodermal activity and decreased heart rate; in contrast, the defensive response facilitates protection from more intense or threatening stimulation and includes both cephalic and digital vasoconstriction, a greater increase in electrodermal activity and increased heart rate $(67,68,70-73)$. The studies reviewed largely missed the chance to facilitate understanding of these constructs, which also may help to explain contradictory or unexpected findings. For example, if the use of a less intense stimulus in some studies elicited an orienting response, the deceleration of heart rate would be the opposite of the expected reaction. Additionally, as the cardiac component of the defensive response may occur over $80 \mathrm{~s}$, with two sequences of heart rate acceleration followed by deceleration, the tendency of many studies to average heart rate over time may have obscured these changes (70).

Design issues: The majority of the studies reviewed failed to distinguish stimulus-specific response patterns by examining painful stimuli alone. Although eight studies did make comparisons with other stimuli, only some comparative stimuli were informative: slides of homicide victims (43), arithmetic and projective test stressors (44), warm pressor $(34,37)$, maximum inspiratory breath hold (61) and vicarious experience (35).

Compared with a relative lack of assessment of stimulus-specific responses, most investigations assessed moderators and mediators of autonomic response to noxious stimulation. Quasiexperimental moderating variables included premenstrual syndrome (41), chronic low back pain (23), trait anxiety $(28,31)$ and exposure to familial pain modelling (66); similarly, experimental manipulation of social influence $(29,49,50)$, hypnosis (40), experimenter sex (4,55), stimulus intensity sequence (42), stimulus predictability (52), imagery-based distraction (28), music engagement (47), nonverbal expression of pain (51), placebo administration $(58,62)$ and scent $(36)$ generally impacted autonomic activity. Finally, studies of mediation provided evidence that autonomic response increases with increased stimulus intensity $(42,56)$, increased report of pain unpleasantness $(57,59)$ and increased attention to the pain stimulus (54).

\section{Measurement and assessment issues}

Given that autonomic response was not always uniform within a single investigation, research measuring only one domain could have missed important findings, and comparisons across studies focusing exclusively on different domains may not be valid. However, 16 studies examined cardiovascular response only, five studies examined pupil dilation only and two studies examined electrodermal activity only. Similarly, most studies assessing cardiac response focused on heart rate, offering little insight into sympathetic versus parasympathetic mediation. The six studies reviewed that included more in-depth cardiac measures indicated both a parasympathetic and a sympathetic influence on cardiac response to pain, although the sympathetic influence predominated $(23,34,55,57,58,62)$. Thus, even if response in a single ANS domain tends to be a more accurate indicator of pain, it may not be sufficient to measure autonomic activity in that domain alone.

The duration of autonomic response measurement also presented a problem for many studies reviewed. Investigations assessing responses for brief, selected durations may have missed patterns of responding occurring outside of measurement periods such as differences in autonomic responding reported in investigations that included poststimulation assessment $(4,23,28,30,33,42,56,62)$. In grouping autonomic responses measured continuously for extended periods into average values without accounting for habituation $(35,43,51,52)$, however, studies could have missed important patterns of responding because other studies offered evidence that habituation occurred in response to repeated stimulation $(23,47,50,53,55)$.

Finally, studies varied in the other modalities of pain responding recorded and analyzed, with the overt motor response of escape assessed in only nine studies $(4,28,29,31,36,38,41,42,53)$. Furthermore, statistical comparisons were made between autonomic and other responses in only 12 studies $(23,33,38,43,45,46,52,54,56-59)$; other investigators simply observed similarities or differences in response patterns. Increased autonomic response was generally related to increased report of pain, but without a priori clarity of theory and hypothesis, post hoc interpretation of results often failed to consider multiple potential relations among modalities. For example, some investigators may interpret increased arousal coupled with increased tolerance as signalling greater acceptance of physiological sensations, but other researchers may anticipate that decreased arousal should accompany lengthened tolerance because they hypothesize that arousal mediates tolerance.

\section{Statistical issues}

Smaller samples can be appropriate when studying a larger effect, but the studies reviewed with smaller sample sizes offered no justification for sample size, which reduces power and impairs interpretation of nonsignificant results. Only six studies reviewed $(4,27,36,38,42,54)$ included a sample of $\geq 74$ participants, and seven studies had groups with as few as 10 participants $(30,33,35,41,49,50,59)$. Furthermore, many researchers presented nonsignificant findings as evidence of no effect, which is faulty logic in a null hypothesis testing paradigm, and only six studies reviewed reported statistics regarding effect size to offer insight into the clinical or practical utility of findings $(27,29,42,47,48,54)$.

Finally, many experiments reviewed included a poor selection of analyses of their data. Only seven studies with repeated measures utilized analyses that addressed sphericity $(23,32,46-48,53,60)$, the frequently violated assumption that correlations between all values of a within-subjects variable will be similar (74-76). Additionally, most investigations inadequately controlled for baseline levels of arousal by analyzing change scores; four studies $(4,28,29,44)$ more properly used analysis of covariance or residualized change scores (12). Discriminant function analysis (43) and causal or mixed-effects modelling $(47,48,54)$, however, offered innovative approaches to analysis.

\section{INTEGRATION OF STUDIES REVIEWED AND CONCLUSIONS}

\section{State of the literature}

The present review is the first formal summary and critique of research investigating autonomic expression of experimentally induced pain in more than 40 years (6). Respiration rate, pupil dilation and electrodermal activity consistently increased during painful stimulation across studies, as well as muscle tension when stimuli were more intense and delivered for a uniform length of time. Cardiovascular responses were more heterogeneous among studies, with neither heart rate nor blood pressure demonstrating consistent responding. Greater precision in measurement revealed more consistent cardiovascular increases in sympathetic activity, with peripheral vasoconstriction and sympathetically driven cardiac responses (eg, heart rate 
variability), although decreased parasympathetic activity did play a role in cardiac responding, consistent with increased respiration rate (12). Additionally, the autonomic component of pain expression was influenced in the expected direction by several variables, including imagery (28), social influence (29), hypnosis (40), engagement with a music listening task (47), attention (54), predictability (52), anxiety (28) and unpleasantness $(57,59)$.

Perhaps most noteworthy regarding the reviewed literature are the numerous barriers to drawing more confident and thorough conclusions; relative to technological innovations, increases in knowledge have not kept pace. Small sample sizes and minimal reporting of effect size cloud interpretation of nonsignificant findings. Studies differed greatly in precision and timing of autonomic assessment, as well as in the individualization or uniformity of stimuli. Many studies failed to address the confounding overlap between painful stimuli and stressful stimuli, which may be expected to elicit similar responding, to illuminate a stimulus-specific ANS pain response. Tentative conclusions suggest decreased skin temperature and increased respiration rate, muscle tension and vasoconstriction may especially characterize autonomic expression of pain $(34,43,61)$. Additionally, although autonomic response typically was related to verbal report, few studies informatively investigated other concordance across response modalities. Considering the disadvantages of the literature, however, the broad conclusions that can be drawn may be quite robust; they also provide a foundation for future research that addresses these limitations.

\section{Conceptual and clinical implications}

The literature regarding pain-induced autonomic arousal has several ramifications. First, painful stimulation produces general autonomic arousal that rises with increasing levels of stimulus intensity, suggesting that autonomic arousal can be assessed as one indicator of pain in research and clinical settings. Not all autonomic responses, however, are equal in relation to pain. Electrodermal or pupillary measures could detect non-pain-specific arousal, and imprecise cardiovascular measures may not be evidence of a pain response. Respiration rate, muscle tension, heart rate variability and peripheral vasoconstriction are more likely to provide a clearer indication of the influence of a painful stimulus.

Second, pain-induced arousal is not simply static; rather, autonomic expression of pain can be modified. Pain management strategies can reduce arousal to decrease the impact of pain on behaviour. Given the importance of respiration, heart rate variability and muscle tension, diaphragmatic breathing or progressive muscle relaxation may be especially effective; however, this assumption requires more direct investigation. Such research has found slowed breathing reduces report of pain (77).

Third, pain remains a construct that must be assessed multimodally. Any conclusions drawn from studies reviewed should not be viewed as support for elevating the veridical status of autonomic responses above verbal report or overt motor behaviour. Collecting measurements from all modalities remains important for assessing all facets of pain sufficiently and comprehensively.

\section{Future directions}

Much work remains to improve understanding of the autonomic response to painful stimulation. First, investigators must use better comparative stimuli to clarify a stimulus-specific autonomic response to painful stimulation. For example, investigations from before the time period covered by the present review compared direct experience of painful stimulation to vicarious, or observational, stimulation, suggesting heart rate accelerates with painful stimulation and decelerates with observation of others receiving painful stimulation (78-80). Similar comparisons are needed in the current literature.

Second, the current literature tends to rely excessively on verbal report; autonomic and other psychophysiological methods (and overt behavioural measures as well) are used much too infrequently. Not only must assessment of pain responding be multimodal, but statistical comparisons must be made among modalities to better elucidate concordance and discordance, rather than simply noting similarities in patterns. Researchers also need to be more specific in the theory organizing their expectation of relations among modalities.

Additionally, differences in methodology across studies, both in administration of painful stimulation and in measurement of autonomic responding, should be better acknowledged and controlled. When possible, it would be ideal to administer stimuli of uniform intensity and duration that are painful to all participants, to balance both internal and external validity, as well as to minimize confounds from differences in participant accuracy, or stimulus duration and intensity. Measures that specifically assess sympathetic and parasympathetic responses will further elucidate the roles of the two branches. Arousal measured over time should be analyzed for trends of shorter duration, as well as patterns of habituation, and more sophisticated statistical analyses should be used to control for individual differences in baseline arousal. Also, to increase power and to help determine if the results found in the studies reviewed apply to other groups, improvements are needed in sample size and demographics; effect sizes must be reported as well.

As a matter of relevance, researchers need to build a stronger case for the relation of experimentally induced pain to acute clinical pain that is either procedural (eg, root canal therapy, tooth extraction) or pathological (eg, kidney stones [nephrolithiasis], arthritis). Better understanding of the relations of autonomic responses to laboratory and naturalistic pain will help illustrate universality of some behavioural principles while revealing factors differentiating contexts.

Because many of the studies reviewed did little to assess or control for cognitive and affective variables frequently associated with pain, these constructs warrant more attention in future research. A significant portion of the pain literature has examined the interaction of fear and anxiety with pain $(81,82)$ That few investigations reviewed included fear or anxiety in their design is especially concerning because these constructs include autonomic arousal as a central feature (11). Also of concern is work to date that blurs the line between fear and anxiety, and pain (eg, using electrical shock to induce fear and the threat of shock to induce anxiety [83]). Similarly, attention is one mechanism through which pain may influence behaviour (84-86); however, the extent to which attention influences the autonomic component of the pain response has been underinvestigated.

To conclude, the literature supports an increase in autonomic response as a component of acute pain, with variations in the response based on the domain of ANS activity measured. Limitations and flaws in the extant literature, however, prevent drawing firm conclusions about a specific ANS response to pain. Tentatively, it may be suggested that electrodermal activity increases and the pupils dilate in response to pain, but respiration rate, muscle tension, heart rate variability and peripheral vasoconstriction are more likely to characterize a unique, signature autonomic response to experimentally induced pain. Future research will offer clarification of the specific nature of this response and how it contributes to researchers' conceptualization of the construct of pain. Perhaps most importantly, concordance between autonomic activity and other response dimensions should not be assumed. Measures in multiple modalities should be included to most sufficiently measure the pain response, to further increase understanding of how autonomic activity fits into the larger picture of laboratoryinduced acute pain. Indeed, measurement of autonomic activity is, and should be, a vital element of pain research.

ACKNOWLEDGEMENTS: The authors express their gratitude to colleagues in the Anxiety, Psychophysiology, and Pain Research Laboratory in the Department of Psychology at West Virginia University (Morgantown, West Virginia, USA). This article is based on a preliminary doctoral examination completed by the first author and supervised by the second author.

FUNDING: Funding for this project was provided by R01-DE014889 (Principal Investigators: Marazita, McNeil, Foxman), and through the Department of Psychology at West Virginia University. 


\section{REFERENCES}

1. International Association for the Study of Pain Task Force on Taxonomy. Part III: Pain terms, a current list with definitions and notes on usage. In: Merskey H, Bogduk, N, eds, Classification of Chronic Pain, 2nd edn. Seattle: IASP Press, 1994:209-14.

2. Gatchel RJ, Epker J. Psychosocial predictors of chronic pain and response to treatment. In: Gatchel RJ, Turk DC, eds. Psychosocial Factors in Pain: Critical Perspectives. New York: Guilford Press, 1999:412-34.

3. Wolff BB. Laboratory methods of pain measurement. In: Melzack R, ed. Pain Measurement and Assessment. New York: Raven Press, 1983:7-13.

4. Carter LE, McNeil DW, Vowles KE, et al. Effects of emotion on pain reports, tolerance and physiology. Pain Res Manag 2002;7:21-30.

5. Feldner MT, Hekmat H. Perceived control over anxiety-related events as a predictor of pain behaviors in a cold pressor task. J Behav Ther Exp Psychiatry 2001;32:191-202.

6. Sternbach RA. Pain: A Psychophysiological Analysis. New York: Academic Press, 1968.

7. Turk DC, Melzack R. The measurement of pain and the assessment of people experiencing pain. In: Turk DC, Melzack R, eds. Handbook of Pain Assessment, 2nd edn. New York: Guilford Press, 2001:3-11.

8. Turk DC, Monarch ES, Williams AD. Assessment of chronic pain sufferers. In: Hadjistavropoulous T, Craig KD, eds. Pain: Psychological Perspectives. Mahwah: Lawrence Erlbaum Associates, 2004:209-43.

9. Wolff BB. Behavioural measurement of human pain. In: Sternbach RA, ed. The Psychology of Pain. New York: Raven Press, 1978:129-68.

10. Cleeland CS. How to treat a 'construct.' J Pain Symptom Manage $1986 ; 1: 161-2$

11. Lang PJ. Fear reduction and fear behavior: Problems in treating a construct. In: Schlien JM, ed. Research in Psychotherapy, Volume 3. Washington, DC: American Psychological Association, 1968:90-102.

12. Larkin KT. Physiological assessment. In: Hersen M, ed. Clinician's Handbook of Adult Behavioral Assessment. Burlington: Academic Press, 2006:165-85.

13. Williams DA. Acute pain (with special emphasis on painful medical procedures). In: Gatchel RJ, Turk DC, eds. Psychosocial Factors in Pain: Critical Perspectives. New York: Guilford Press, 1999:151-63.

14. Stern RM, Ray WJ, Quigley KS. Psychophysiological Recording, 2nd edn. New York: Oxford University Press, 2001.

15. Task Force of the European Society of Pacing and Electrophysiology. Heart rate variability standards of measurement, physiological interpretation, and clinical use. Circulation 1996;93:1043-65.

16. Gibson SJ, Chambers CT. Pain over the life span: A developmental perspective. In: Hadjistavropoulous T, Craig KD, eds. Pain: Psychological Perspectives. Mahwah: Lawrence Erlbaum Associates, 2004:113-53.

17. Flor H. Psychophysiological assessment of the patient with chronic pain. In: Turk DC, Melzack R, eds. Handbook of Pain Assessment, 2nd edn. New York: Guilford Press, 2001:76-96.

18. Benarroch EE. Pain-autonomic interactions: A selective review. Clin Auton Res 2001;11:343-9.

19. Price DD. Central neural mechanisms that interrelate sensory and affective dimensions of pain. Mol Interv 2002;2:392-402.

20. Willis WD, Westlund KN. Neuroanatomy of the pain systems and of the pathways that modulate pain. J Clin Neurophysiol 1997;14:2-31.

21. Wilder J. Modern psychophysiology and the law of initial value. Am J Psychother 1958;12:199-221.

22. Sweet SD, McGrath PJ. Physiological measures of pain. In: Finley GA, McGrath PJ, eds. Progress in Pain Research and Management: Volume 10. Measurement of Pain in Infants and Children. Seattle: IASP Press, 1998:59-81.

23. Peters ML, Schmidt AJ. Psychophysiological responses to repeated acute pain stimulation in chronic low back pain patients. J Psychosom Res 1991;35:59-74.

24. Dougher MJ, Goldstein D, Leight KA. Induced anxiety and pain. J Anxiety Disord 1987;1:259-64.

25. Forgione A, Barber TX. A strain gauge pain stimulator. Psychophysiology 1971;8:102-6.

26. Rainwater AJ, III, McNeil DW. Reinventing the algometer: Synopsis of the literature and presentation of a reliable, inexpensive model. Behav Res Methods Instruments Comput 1991;23:486-92.
27. Foster RL, Yucha CB, Zuk, J, Vojir CP. Physiologic correlates of comfort in healthy children. Pain Manage Nurs 2003;4:23-30.

28. Biederman JJ, Schefft BK. Behavioral, physiological, and self-evaluative effects of anxiety on the self-control of pain. Behav Modif 1994;18:89-105.

29. Platow MJ, Voudouris NJ, Coulson M, et al. In-group reassurance in a pain setting produces lower levels of physiological arousal: Direct support for a self-categorization analysis of social influence. Eur J Soc Psychol 2007;37:649-60.

30. Feuerstein M, Barr RG, Francoeur TE, Houle M, Rafman S. Potential biobehavioral mechanisms of recurrent abdominal pain in children. Pain 1982;13:287-98.

31. James JE, Hardardottir D. Influence of attention focus and trait anxiety on tolerance of acute pain. Br J Health Psychol 2002;7:149-62.

32. Peckerman A, Saab PG, McCabe PM, et al. Blood pressure reactivity and perception of pain during the forehead cold pressor test. Psychophysiology 1991;28:485-95.

33. Tassorelli C, Micieli G, Osipova V, Rossi F, Nappi G. Pupillary and cardiovascular responses to the cold-pressor test. J Auton Nerv Syst 1995;55:45-9.

34. Tomaka J, Blascovich J, Kibler J, Ernst JM. Cognitive and physiological antecedents of threat and challenge appraisal. J Pers Soc Psychol 1997;73:63-72.

35. Yamaguchi S, Harano K, Egawa B. Effects of differentially modeled stimuli on vicarious autonomic arousal. Percept Mot Skills 1978;46:643-50.

36. Raudenbush B, Koon J, Meyer B, Corley N, Flower N. Effects of odorant administration on pain and psychophysiological measures in humans. N Am J Psychol 2004;6:361-70.

37. Flora SR, Wilkerson LR, Flora DB. Effects of cold pressor pain on human self-control for positive reinforcement. Psychol Rec 2003;53:243-52.

38. Myers CD, Robinson ME, Riley JL, Sheffield D. Sex, gender, and blood pressure: Contributions to experimental pain report. Psychosom Med 2001;63:545-50.

39. Zeichner A, Loftin M, Panopoulos G, Widner S, Allen J. Sex differences in pain indices, exercise, and use of analgesics. Psychol Rep 2000;86:129-33.

40. Walter H, Lesch OW, Stöhr H, Grünberger J, Gutierrez-Lobos K. Reaction to a pain stimulus before and during hypnosis measured by pupillary reaction. Am J Clin Hypnosis 2005;48:145-52.

41. Kuczmierczyk AR, Adams HE. Autonomic arousal and pain sensitivity in women with premenstrual syndrome at different phases of the menstrual cycle. J Psychosom Res 1986;30:421-8.

42. Kyle BN, McNeil DW, Weinstein BJ, Mark JD. Interaction of intensity and order regarding painful events. J Behav Med 2009;32:360-70.

43. Davidson PO, Neufeld RW. Response to pain and stress: A multivariate analysis. J Psychosom Res 1974;18:25-32.

44. Linden W. Cardiovascular response as a function of predisposition, coping behavior and stimulus type. J Psychosom Res 1985;29:611-20.

45. Ellermeier W, Westphal W. Gender differences in pain ratings and pupil reactions to painful pressure stimuli. Pain 1995;61:435-9.

46. Höfle M, Kenntner-Mabiala R, Pauli P, Alpers GW. You can see pain in the eye: Pupillometry as an index of pain intensity under different luminance conditions. Int J Psychphysiol 2008;70:171-5.

47. Bradshaw DH, Chapman CR, Jacobson RC, Donaldson GW. Effects of music engagement on responses to painful stimulation. Clin J Pain 2012;28:418-27.

48. Chapman CR, Oka S, Bradshaw DH, Jacobson RC, Donaldson GW. Phasic pupil dilation response to noxious stimulation in normal volunteers: Relationship to brain evoked potentials and pain report. Psychophysiology 1999;36:44-52.

49. Craig KD, Neidermayer H. Autonomic correlates of pain thresholds influenced by social modeling. J Pers Soc Psychol 1974;29:246-52.

50. Craig KD, Prkachin KM. Social modeling influences on sensory decision theory and psychophysiological indexes of pain. J Pers Soc Psychol 1978;36:805-15.

51. Lanzetta JT, Cartwright-Smith J, Kleck RE. Effects of nonverbal dissimulation on emotional experience and autonomic arousal. J Pers Soc Psychol 1976;33:354-70.

52. Lykken DT, Macindoe I, Tellegen A. Preception: Autonomic response to shock as a function of predictability in time and locus. Psychophysiology 1972;9:318-33. 
53. Vassend O, Knardahl S. Effects of repeated electrocutaneous pain stimulation on facial blood flow. Biol Psychol 2005;68:163-78.

54. Chapman CR, Donaldson DW, Nakamura Y, Jacobson RC, Bradshaw DH, Gavrin J. A psychophysiological causal model of pain report validity. J Pain 2002:3:143-55.

55. Aslaksen PM, Myrbakk IN, Høifødt RS, Flaten MA. The effect of experimenter gender on autonomic and subjective responses to pain stimuli. Pain 2007;129:260-8.

56. Möltner A, Hölzl R, Strian F. Heart rate changes as an autonomic component of the pain response. Pain 1990;43:81-9.

57. Tousignant-Laflamme Y, Rainville P, Marchand S. Establishing a link between heart rate and pain in healthy subjects: A gender effect. J Pain 2005;6:341-7.

58. Aslaksen PM, Flaten MA. The roles of physiological and subjective stress in the effectiveness of a placebo on experimentally induced pain. Psychosom Med 2008;70:811-8.

59. Rainville P, Carrier B, Hofbauer RK, Bushnell MC, Duncan GH. Dissociation of sensory and affective dimensions of pain using hypnotic modulation. Pain 1999;82:159-71.

60. Porro CA, Cettolo V, Francescato MP, Baraldi P. Functional activity mapping of the mesial hemispheric wall during anticipation of pain. NeuroImage 2003;19:1738-47.

61. Kimura Y, Ge HY, Zhang Y, Kimura M, Sumikura H, Arendt-Nielsen L. Evaluation of sympathetic vasoconstrictor response following nociceptive stimulation of latent myofascial trigger points in humans. Acta Physiol 2009;196:411-7.

62. Pollo A, Vighetti S, Rainero I, Benedetti F. Placebo analgesia and the heart. Pain 2003;102:125-33.

63. Keppel G, Wickens TD. Experimental design. In: Keppel G, Wickens TD, eds. Design and Analysis: A Researcher's Handbook, 4th edn. Upper Saddle River: Prentice Hall, 2004:2-11.

64. Maxwell SE. The persistence of underpowered studies in psychological research: Causes, consequences, and remedies. Psychol Methods 2004;9:147-63.

65. Riley JL, Robinson ME, Wise EA, Myers CD, Fillingim RB. Sex differences in the perception of noxious experimental stimuli: A meta-analysis. Pain 1998;74:181-7.

66. Zeichner A, Widner S, Loftin M, Panopoulos G, Allen J. Effects of familial pain models on daily pain indices and performance during the cold pressor task. Psychol Rep 1999;84:955-60.

67. Andreassi JL. Psychophysiology: Human Behavior \& Physiological Response. Mahwah: Lawrence Erlbaum Associates, 2007.

68. Turpin G. Effects of stimulus intensity on autonomic responding: The problem of differentiating orienting and defense reflexes. Psychophysiology 1986;23:1-14.

69. Turpin G, Schaefer F, Boucsein W. Effects of stimulus intensity, risetime, and duration on autonomic and behavioral responding: Implications for the differentiation of orienting, startle, and defense responses. Psychophysiology 1999;36:453-63.
70. Vila J, Guerra P, Muñoz MA, et al. Cardiac defense: From attention to action. Int J Psychophysiol 2007;66:169-82.

71. Balaban MT, Rhodes DL, Neuringer A. Orienting and defense responses to punishment: Effects on learning. Biol Psychol 1990;30:203-17.

72. Graham FK, Clifton RK. Heart-rate change as a component of the orienting response. Psychol Bull 1966;65:305-20.

73. Sokolov YN. Perception and the Conditioned Reflex. New York: Macmillan, 1963.

74. Keppel G, Wickens TD. The single-factor within-subject design: Further topics. In: Keppel G, Wickens TD, eds. Design and Analysis: A Researcher's Handbook, 4th edn. Upper Saddle River: Prentice Hall, 2004:369-400.

75. Tabachnick BG, Fidell LS. Profile analysis: The multivariate approach to repeated measures. In: Tabachnick BG, Fidell LS, eds. Using Multivariate Statistics, 5th edn. Boston: Pearson Education, 2007:311-74.

76. Weinfurt KP. Repeated measures analyses: ANOVA, MANOVA, and HLM. In: Grimm LG, Yarnold PR, eds. Reading and Understanding More Multivariate Statistics. Washington, DC: American Psychological Association, 2000:317-61.

77. Martin SL, Kerr, KL, Bartley EJ, et al. Respiration induced hypoalgesia: Exploration of potential mechanisms. J Pain 2012;13:755-63.

78. Craig KD. Physiological arousal as a function of imagined, vicarious and direct stress experiences. J Abnorm Psychol 1968;73:513-20.

79. Craig KD, Lowery HJ. Heart-rate components of conditioned vicarious autonomic responses. J Pers Soc Psychol 1969;11:381-7.

80. Craig KD, Wood K. Physiological differentiation of direct and vicarious affective arousal. Can J Behav Sci 1969;1:98-105.

81. McNeil DW, Rainwater AJ. Development of the Fear of Pain Questionnaire-III. J Behav Med 1998;21:389-410.

82. Vowles KE, McNeil DW, Sorrell JT, Lawrence SM. Fear and pain: Investigating the interaction between aversive states. J Abnorm Psychol 2006;115:821-33.

83. Rhudy JL, Meagher MW. Fear and anxiety: Divergent effects on human pain thresholds. Pain 2000;84:65-75.

84. Eccleston C. Chronic pain and attention: A cognitive approach. Br J Clin Psychol 1994;33:535-47.

85. Eccleston C. Chronic pain and distraction: An experimental investigation into the role of sustained and shifting attention in the processing of chronic persistent pain. Behav Res Ther 1995;33:391-405.

86. Eccleston C, Crombez G. Pain demands attention: A cognitiveaffective model of the interruptive function of pain. Psychol Bull 1999;125:356-66. 


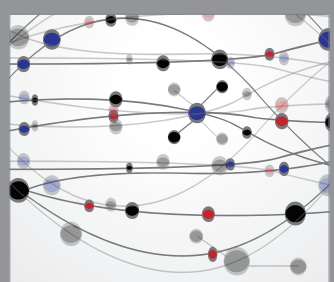

The Scientific World Journal




Gastroenterology Research and Practice



\section{Hindawi}

Submit your manuscripts at

http://www.hindawi.com
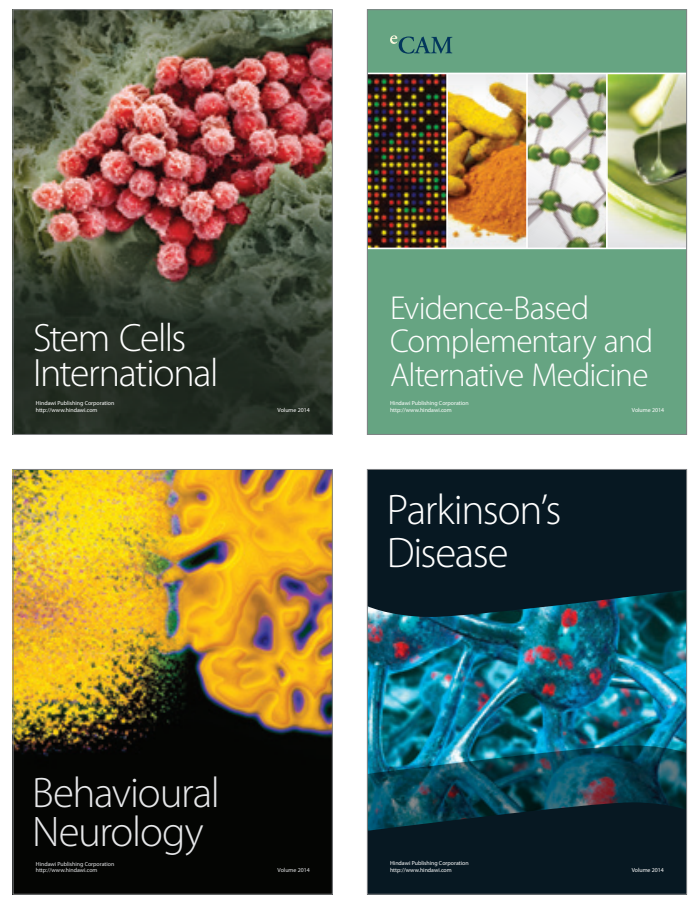
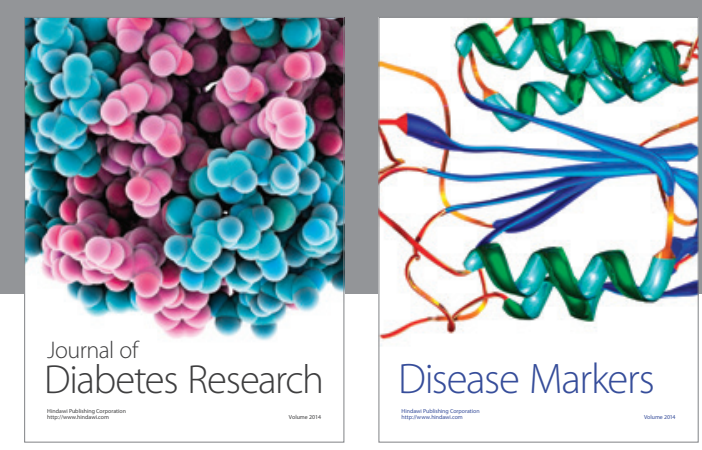

Disease Markers
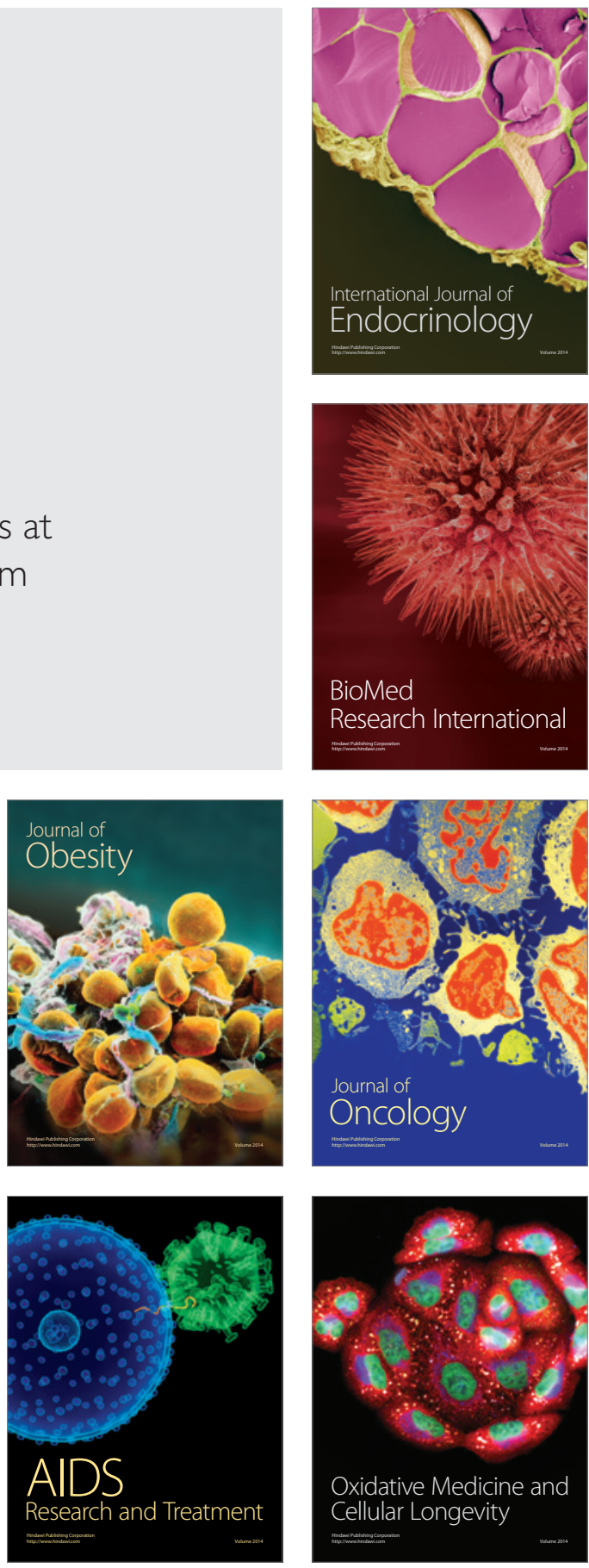